\title{
Role of Oculoproprioception in Coding the Locus of Attention
}

\author{
Bartholomaeus Odoj $^{1,2}$ and Daniela Balslev ${ }^{3}$
}

\begin{abstract}
The most common neural representations for spatial attention encode locations retinotopically, relative to center of gaze. To keep track of visual objects across saccades or to orient toward sounds, retinotopic representations must be combined with information about the rotation of one's own eyes in the orbits. Although gaze input is critical for a correct allocation of attention, the source of this input has so far remained unidentified. Two main signals are available: corollary discharge (copy of oculomotor command) and oculoproprioception (feedback from extraocular muscles). Here we asked whether the oculoproprioceptive signal relayed from the somatosensory cortex contributes to coding the locus of attention. We used continuous theta burst stimulation (cTBS) over a human oculoproprioceptive area in the postcentral
\end{abstract}

\section{INTRODUCTION}

Attention allows organisms to focus on relevant stimuli. In monkeys, neurons that respond to attended stimuli have a retinotopic receptive field, which reference location relative to the direction of gaze (Gottlieb, Kusunoki, \& Goldberg, 1998; Andersen \& Mountcastle, 1983). A retinotopic code for attended stimuli has also been observed in humans. Indeed, although visual perception is normally facilitated at the location indicated by an attention cue, just after an eye movement, attention can be transiently allocated away from the cue, at its previous retinotopic coordinates (Talsma, White, Mathôt, Munoz, \& Theeuwes, 2013; Golomb, Nguyen-Phuc, Mazer, McCarthy, \& Chun, 2010; Mathôt \& Theeuwes, 2010; Golomb, Chun, \& Mazer, 2008).

Retinotopic representations alone cannot support cross-modal interactions in spatial attention between visual and nonvisual modalities or maintain a stable focus of attention across eye movements. One solution to this problem could be to create coregistered representations in multiple reference frames (Pouget, Deneve, \& Duhamel, 2002; Andersen, Snyder, Bradley, \& Xing, 1997). Another solution could be to update the retinotopic representation for each eye movement (Duhamel, Colby, \& Goldberg,

${ }^{1}$ University of Tübingen, ${ }^{2}$ University of Copenhagen, ${ }^{3}$ University of St. Andrews gyrus $\left(\mathrm{S} 1_{\mathrm{EYE}}\right) . \mathrm{S} 1_{\mathrm{EYE}^{-}} \mathrm{c}$ TBS reduces proprioceptive processing, causing $\sim 1^{\circ}$ underestimation of gaze angle. Participants discriminated visual targets whose location was cued in a nonvisual modality. Throughout the visual space, $\mathrm{S} 1_{\mathrm{EYE}}$-CTBS shifted the locus of attention away from the cue by $\sim 1^{\circ}$, in the same direction and by the same magnitude as the oculoproprioceptive bias. This systematic shift cannot be attributed to visual mislocalization. Accuracy of open-loop pointing to the same visual targets, a function thought to rely mainly on the corollary discharge, was unchanged. We argue that oculoproprioception is selective for attention maps. By identifying a potential substrate for the coupling between eye and attention, this study contributes to the theoretical models for spatial attention.
1992). Importantly, both solutions require information about the rotation of the eyes in the orbits. Despite the importance of the gaze information in the brain's representations for spatial attention, the sources of this gaze input to the attention maps remain unknown. The main sources of eye position are the feedback from the extraocular muscles or oculoproprioception (Sherrington, 1918) and an internal model that predicts future eye rotation based on the copy of the oculomotor command or corollary discharge (von Helmholtz, 1925).

Does oculoproprioception play a role in coding the locus of attention? On the one hand, some studies fail to find a decrease in accuracy or precision of visual localization in conditions when oculoproprioception is reduced or abnormal (Balslev, Himmelbach, Karnath, Borchers, \& Odoj, 2012; Lewis, Gaymard, \& Tamargo, 1998; Guthrie, Porter, \& Sparks, 1983). Such findings support the suggestion that oculoproprioception does not normally contribute to the estimate of eye rotation but rather calibrates the oculomotor command in the long term (Wurtz, 2008; Steinbach, 1986).

On the other hand, some behavioral studies (Talsma et al., 2013; Golomb et al., 2008, 2010; Mathôt \& Theeuwes, 2010) show delays in updating the retinotopic coordinates where attention is deployed after a saccade. These delays are compatible with the delay in the ascending fibers (Xu, Wang, Peck, \& Goldberg, 2011; Wang, Zhang, Cohen, \& 
Goldberg, 2007) and therefore leave open the possibility that oculoproprioception may contribute. Furthermore, we have observed alterations in visual sensitivity in conditions that distort the oculoproprioceptive signal. The oculoproprioceptive signal was distorted in the ocular periphery, using passive eye rotation (Balslev, Newman, \& Knox, 2012), or centrally, in the somatosensory cortex, after repetitive TMS (Odoj \& Balslev, 2013; Balslev, Gowen, \& Miall, 2011) or after a focal lesion (Balslev, Odoj, \& Karnath, 2013). These previous studies suggest a link between the oculoproprioceptive signal in the somatosensory cortex and spatial attention; however, the nature of this link is still unclear. One possibility is that the attention map incorporates oculoproprioception, so that a distortion of this signal causes a systematic shift in the locus of attention relative to a cue. Another possibility is that the visual map incoporates oculoproprioception, so that a distortion of this signal causes a systematic error in locating visual targets relative to the body. Visual stimuli presented nearer the hand (Reed, Grubb, \& Steele, 2006), the head midline (Durand, Camors, Trotter, \& Celebrini, 2012; Durand, Trotter, \& Celebrini, 2010), or the trunk midline (Grubb, Reed, Bate, Garza, \& Roberts, 2008) have a privileged access to neural processing resources compared with visual stimuli presented elsewhere. A mislocalization of the visual stimuli relative to these landmarks could, in our previous studies, have changed their neural processing priority.

Here, we investigated whether oculoproprioception is incorporated in attention maps.

Oculoproprioception was manipulated using continuous theta burst stimulation (CTBS), a form of inhibitory TMS, over an oculoproprioceptive area in the human postcentral gyrus ( $\left(\mathrm{S}_{\mathrm{EYE}}\right)$. Inhibitory repetitive TMS over $\mathrm{S} 1_{\mathrm{EYE}}$ interferes with the ability to correct for passive eye movement during a visual localization task (Balslev \& Miall, 2008) and results in an underestimation of the rotation of the eye in the orbit by $\sim 1^{\circ}$ (Odoj \& Balslev, 2013). We used $S 1_{\mathrm{EYE}}$-CTBS over the left postcentral gyrus to alter the oculoproprioceptive signal. Participants discriminated visual targets whose location was cued by the position of their unseen left index finger or pointed to these targets using the same finger. Both tasks depend critically on eye position information to match the location of the visual target with the location of the finger that acted either as an attention cue or as an effector.

We report a systematic error in the locus of attention and no pointing error after $S 1_{\mathrm{EYE}}-\mathrm{CTBS}$. We argue therefore that oculoproprioception is the eye position signal that is selective for the attention map.

\section{METHODS}

\section{Overview of the Experiments}

Experiment 1 examined the allocation of attention using a cross-modal task. Participants discriminated a visual target. The location of this target was cued by the location of the unseen left index finger. The locus of attention was defined as the location in the visual space where the cue had the largest effect on the RT for target discrimination. If oculoproprioception is incorporated into the attention maps, one would predict a systematic error in the locus of attention relative to the location of the cue after S1 $1_{\mathrm{EYE}}$-CTBS.

Experiment 2 examined visuospatial localization using an open-loop pointing task. Participants used their unseen left index finger to reach to visual targets in the absence of visual feedback. If oculoproprioception is incorporated into visual maps, one would predict a systematic error in open-loop pointing after $\mathrm{S} 1_{\mathrm{EYE}}$-CTBS.

Experiment 3 examined participants' ability to locate their unseen left index finger using an open-loop pointing task. This experiment controlled for an effect of $S 1_{\mathrm{EYE}}$-CTBS on the ability to locate the nonvisual cue in Experiment 1.

All experiments were conducted with the participants fixating rightward (Experiments $1 \mathrm{~A}-3 \mathrm{~A})$ and leftward (Experiments $1 \mathrm{~B}-3 \mathrm{~B})$. The reason for repeating the experiment for different directions of gaze was the following. The effect of $S 1_{\mathrm{EYE}}$-cTBS on visual localization is gaze dependent, that is, a shift in perceived eye rotation toward left in rightward gaze and toward right in leftward gaze (Odoj \& Balslev, 2013), reflecting the underestimation of the rotation of the eyes in the orbits. We predicted therefore that the change in the direction of gaze would reverse the effect. In this way, one can separate a specific, gaze-dependent effect, from a general influence of anterior parietal cortex on attention (Experiment 1) or reaching (Experiments 2-3).

\section{Participants}

We tested 10 participants (five women) in each of three different experiments (Experiments 1-3). All were righthanded with normal or corrected-to-normal vision. For Experiments 1A-3A, participants had an age range from 22 to 32 years (median: 28.5 years). For Experiments 1B-3B, participants had an age range from 27 to 32 years (median: 29 years). Five participants took part in all experiments (A and B). All participants gave their informed consent. The study was approved by the local ethics committee at the University of Tübingen.

\section{Experiment 1. Attention Map}

This experiment investigated whether oculoproprioception is included in the eye position estimate necessary for orienting attention in the visual space in response to a nonvisual cue.

Participants discriminated a target (letter "A" or " $\mathrm{H}$ ", size $1^{\circ}$ visual angle) whose most probable location was indicated by a somatosensory cue, the location of the participant's left index finger hidden from view. We assumed that cTBS of the ipsilateral, left somatosensory cortex will have 
only a small effect, if any on perceiving finger location. This assumption was explicitly tested in Experiment 2. The experimenter placed the participant's index finger just below the horizontal line where targets were presented. To assess the benefit of the cue, we calculated the difference in RT for visual discrimination in the presence versus the absence of the cue. The locus of attention was the location with the largest benefit of the cue. "Cueing error" was defined as the distance between the locus of attention and the actual location of the cue. If eye proprioception contributes to coding the locus of attention, $\mathrm{S} 1_{\mathrm{EYE}}$-CTBS should increase cueing error by causing a shift in the locus of attention away from the cue and toward the center of the orbit, in the same direction as the shift in perceived eye position.

\section{Setup}

Participants sat with their head fixed in a chin rest and cheek pads. A cathode ray tube (CRT) display was placed at $45 \mathrm{~cm}$ in front of them (Figure 1A). The CRT was centered at $+19^{\circ}$ (Experiment 1A) or at $-19^{\circ}$ (Experiment $1 \mathrm{~B}$, Figure 1) from center of the right orbit. Participants performed the task in right eye vision, with the left eye patched. The experiment was conducted in monocular vision for the following reason. In the macaque, the primary oculoproprioceptive area 3 a receives proprioceptive information from the contralateral eye only (Wang et al., 2007). In humans, although proprioceptive information from both eyes is relayed to each brain hemisphere (Balslev, Albert, \& Miall, 2011), the input from the contralateral eye is functionally more important (Balslev, Himmelbach, et al., 2012). Therefore, we assumed that after cTBS of one hemisphere (left), the effects would be strongest and easier to measure for the contralateral eye (right). As a somatosensory cue, we used the position of the participants' left index finger, ipsilateral to the hemisphere where TMS was applied. A transparent (Plexiglas) sheet was mounted $5 \mathrm{~cm}$ in front of the CRT screen. Participants had their left index finger on a support (a wooden ledge) attached to the Plexiglas immediately under the location where the targets would appear. The finger was placed at one of four possible cue positions, $8^{\circ}, 18^{\circ}, 20^{\circ}$, or $30^{\circ}$ to the right (Experiment $1 \mathrm{~A}$, rightward gaze) or left (Experiment $1 \mathrm{~B}$, leftward gaze) from orbit midline of the right eye and covered with black cloth by the experimenter. The experiment took place in total darkness. The participants had no visual information about finger location (somatosensory cue) to prompt the use of gaze information to locate the visual targets relative to the cue.

At the beginning of the trial, participants fixated on a central cross (white, $1^{\circ} \times 1^{\circ}$ ) presented on black background (Figure 1B). Fixation was verified with a headmounted eye tracker (EyeLink II, SR Research Ltd., Ottawa, Canada). After 500-650 msec (randomized), the fixation cross disappeared. At $100 \mathrm{msec}$ later, a target letter ("A" or "H," $1^{\circ}$ visual angle) appeared for $100 \mathrm{msec}$. The target appeared at one of seven possible locations, at $-3^{\circ},-2^{\circ}$, $-1^{\circ}, 0^{\circ}, 1^{\circ}, 2^{\circ}$, and $3^{\circ}$ from the somatosensory cue. Participants were told that the target letter is most likely to appear at the location indicated by the cue. The target letter was however presented with equal probability (eight times) at each of the seven possible target locations. Additionally, three trials showed target letters at random locations outside this range so that the participants could not predict the location of the nonvisual cue from the spatial distribution of the visual targets. The participants were instructed to name the target letter as fast and accurately as possible. Voice RT was recorded.

Trials with the same cue location were grouped in blocks. Each block consisted of 59 trials ( 8 trials for each of the 7 target positions +3 random positions). Trial order was pseudorandomized. At the end of each block, participants were instructed to close their eyes. Then the experimenter moved the participants' index finger at the next cue location and started a new block. The participants completed four cued blocks (cue at $+8^{\circ},+18^{\circ}$,
Figure 1. Setup and task for the cross-modal attention experiment. (A) In right eye vision, participants fixated at $+19^{\circ}$ (Experiment 1A, rightward gaze) or $-19^{\circ}$ (Experiment $1 \mathrm{~B}$, leftward gaze) from the center of the right orbit. A somatosensory cue (the participants' left index finger, hidden from view) was positioned at one of four possible locations: $+8^{\circ},+18^{\circ}$, $+20^{\circ}$, or $+30^{\circ}$ (Experiment $\left.1 \mathrm{~A}\right)$ or $-8^{\circ},-18^{\circ},-20^{\circ}$, or $-30^{\circ}$ (Experiment 1B) from orbit midline (here, Experiment $1 \mathrm{~B}$ : fixation at $-19^{\circ}$, cue at $-30^{\circ}$ from orbit midline). (B) A target letter, "A" or "H," was presented for $100 \mathrm{msec}$ at one of seven possible locations, at $-3^{\circ},-2^{\circ},-1^{\circ}, 0,+1^{\circ},+2^{\circ}$, and $+3^{\circ}$ horizontally from the cue. Participants named the letter as fast and correct as possible. Voice RT and accuracy were recorded. The solid line shows eye position at fixation. The dotted line shows perceived eye position after $\mathrm{S} 1_{\mathrm{EYE}}$-CTBS according to Odoj and Balslev (2013). We predicted a shift of the locus of attention congruent to the shift in perceived gaze toward the center of the orbit for all cue locations. 
$+20^{\circ}$ or $+30^{\circ}$ from orbit midline in Experiment $1 \mathrm{~A}$ and $-8^{\circ},-18^{\circ},-20^{\circ}$ or $-30^{\circ}$ in Experiment 1B). The order of the blocks was pseudorandomized.

To assess the baseline distribution of attention as well as visual accuracy, participants performed the same visual discrimination task in the absence of a cue. Participants' left index finger rested in front of their body midline. Target letters were presented on the screen at all locations tested in the cued blocks. These locations were probed in random order, four times each. The uncued block consisted of 92 trials. This block was performed either before or after the cued blocks, randomized across participants.

\section{Data Analysis}

We calculated the difference in RT for visual discrimination in the presence versus the absence of the cue. The locus of attention was the location with the largest benefit of the cue (the largest decrease in RT in the presence vs. the absence of the cue). The cueing error was calculated as the distance between the locus of attention and the location of the cue (Figure 2).

Cueing error for each cue location was compared before and after cTBS using paired-samples $t$ tests. Mean cueing error was analyzed using a repeated-measures ANOVA with factors (1) TMS run (pre vs. post), (2) Stimulation area (S1 $1_{\mathrm{EYE}}$ vs. $\mathrm{P} 3$ ), and (3) Gaze direction (leftward vs. rightward). If oculoproprioception is incorporated into attention maps, one would predict a significant threeway interaction, driven by an increased cueing error after $\mathrm{S} 1_{\mathrm{EYE}} \mathrm{C}^{\mathrm{CTBS}}$. The cueing error was expected to have oppo-

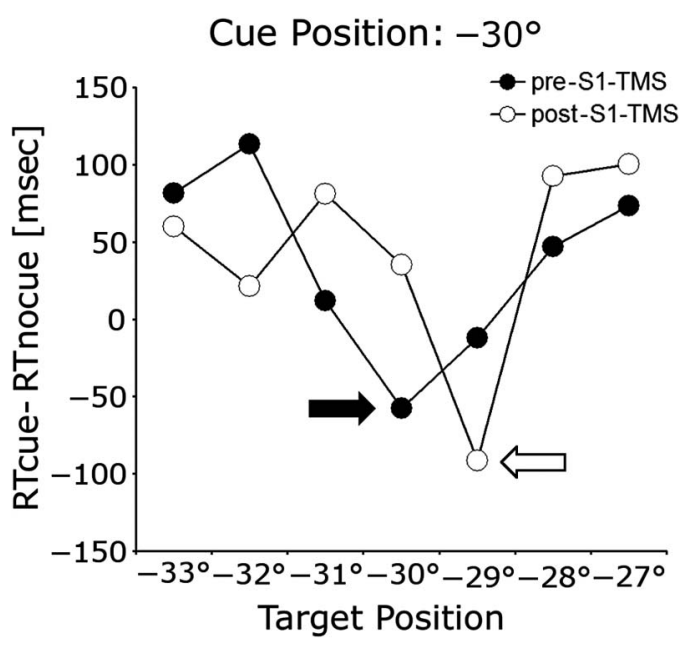

Figure 2. Cueing error in one example participant with a somatosensory cue at $-30^{\circ}$ from orbit midline, before and after $\mathrm{S} 1_{\mathrm{EYE}}$-cTBS. Before CTBS over $\mathrm{S} 1_{\mathrm{EYE}}(-)$, the largest decrease in $\mathrm{RT}$ in the presence versus in the absence of the cue occurred for the target that appeared at the same location as the cue (black arrow, cueing error $=0^{\circ}$ ). After $\mathrm{S} 1_{\mathrm{EYE}}$-cTBS (O), the largest benefit of the cue was observed for the target that appeared at $1^{\circ}$ toward the midline (white arrow). site sign for leftward and rightward gaze, mirroring the underestimation of the angle of gaze after $\mathrm{S} 1_{\mathrm{EYE}^{-C}} \mathrm{TBS}$ (Experiment 1 in Odoj \& Balslev, 2013).

To assess whether the results are robust, we repeated the analysis using a different method for calculating the locus of attention. We now defined the locus of attention as the center of mass of all locations that showed a cueing benefit. First, we identified all locations showing a benefit of the cue, indicated by a faster RT with the cue than without the cue. Then, we calculated the mean of these locations after weighting each location with the magnitude of the cueing effect there. To separate out an eventual practice effect (i.e., an improvement of RT that was common to all target locations within a block), we preprocessed the data by subtracting the mean RT for each block of trials.

\section{Experiment 2. Visual Map}

Experiment 2 examined the ability to locate visual targets relative to the left index finger after $\mathrm{S} 1_{\mathrm{EYE}}$-CTBS using a pointing task. This experiment also controlled for an error in hand proprioception after cTBS in the postcentral gyrus, to rule out a systematic error in the perception of the nonvisual cue in Experiment 1.

Participants pointed with their left index finger, the same finger that was used as a nonvisual cue in Experiment 1 . Visual targets were presented at the same locations as the cues in Experiment 1. If locating visual targets for reaching takes oculoproprioception into account or if cTBS of the left postcentral gyrus alters perceived posture of the ipsilateral hand, one would expect pointing errors after $\mathrm{S} 1_{\mathrm{EYE}}$-CTBS.

\section{Setup}

The setup was identical with that used in Experiment 1. Additionally, a position sensor (Polhemus Fastrak, Colchester, VT) was fixed on the tip of the participants left index finger.

At the beginning of the trial, the participants fixated on a central cross $\left(1^{\circ} \times 1^{\circ}\right.$, white on black background). Fixation was verified with the head-mounted eye tracker. Participants' left index finger rested in front of their body midline (Figure 3A). After 500-650 msec (randomized), the fixation cross disappeared. At $100 \mathrm{msec}$ later, a target letter ("X") appeared for $100 \mathrm{msec}$. The target could appear at four possible locations: at $8^{\circ}, 18^{\circ}, 20^{\circ}$, or $30^{\circ}$ from orbit midline in Experiment $2 \mathrm{~A}$ or at $-8^{\circ},-18^{\circ},-20^{\circ}$, or $-30^{\circ}$ from orbit midline in Experiment $2 \mathrm{~B}$. The participants were instructed to close their eyes, then point with their left index finger as accurately as possible at the remembered location of the target. The reaching movement stopped on the wooden ledge when the finger touched the plexiglass. Participants were allowed to adjust the position of their finger until they felt the finger was pointing to the target. After participants confirmed that this was the case, finger position was recorded, and the experimenter moved the finger back to the 
Figure 3. Setup and task for the visual open-loop pointing experiment. (A) Participants fixated at $+19^{\circ}$ (Experiment 2A) or $-19^{\circ}$ (Experiment $2 \mathrm{~B}$ ) from the center of the orbit, while a position marker was attached to their left index finger (here: fixation at $-19^{\circ}$ ). (B) A target (letter "X") was presented for $100 \mathrm{msec}$ at one of four possible positions: $+8^{\circ},+18^{\circ},+20^{\circ}$,

$+30^{\circ}$ from orbit midline (Experiment $2 \mathrm{~A}$ ) or $-8^{\circ},-18^{\circ}$,

$-20^{\circ},-30^{\circ}$ from orbit midline (Experiment $2 \mathrm{~B}$ ). After target presentation, participants closed their eyes and pointed to the location where the target appeared. Finger location was recorded, and then the finger was passively moved back to the resting position. The solid line shows eye position at fixation. The dotted line shows perceived gaze direction after S1 $1_{\mathrm{EYE}}$-CTBS according to Odoj and Balslev (2013).

resting position at body-midline. Trials for each target position were grouped in blocks. Each block consisted of six trials. Block order was pseudorandomized.

\section{Data Analysis}

Pointing error was calculated as the signed distance between target and finger location at the end of the movement. Pointing error for each target location was compared before and after cTBS using paired-samples $t$ tests. Mean pointing error across all target locations was analyzed using a repeated-measures ANOVA with factors (1) TMS run (pre vs. post), (2) Stimulation area (S1 $1_{\mathrm{EYE}}$ vs. $\mathrm{P} 3$ ), and (3) Gaze direction (leftward vs. rightward). If oculoproprioception is incorporated into visual maps, one would predict a significant three-way interaction, driven by an increased pointing error after $\mathrm{S} 1_{\mathrm{EYE}}$-CTBS. The pointing error was expected to have opposite sign for leftward and rightward gaze, mirroring the underestimation of the angle of gaze after $\mathrm{S}_{\mathrm{EYE}^{-}} \mathrm{CTBS}$ (Experiment 1 in Odoj \& Balslev, 2013).

\section{Experiment 3. Perceived Finger Posture}

Experiment 1 required to match the position of the unseen finger at rest with a visual target, whereas Experiment 2 asked participants to point the same finger at a visual target without visual feedback. One could explain a systematic cueing error in Experiment 1 despite accurate open-loop pointing in Experiment 2 by an error in the perceived posture of the left index finger at rest. To investigate whether $\mathrm{S} 1_{\mathrm{EYE}}$-CTBS selectively disturbs the felt position of the left index finger at rest (rather than during movement), we conducted a third experiment. In this experiment, participants were asked to point with the left index finger to the remembered position of this finger. The participants had their eyes closed and thus had no visual feedback. We measured pointing error as the difference between the location of finger at the end of movement and the location where the left index finger was passively placed.
The setup was identical with Experiment 2. At the beginning of each trial, the experimenter took the participant's left index finger from the starting position in front of the body-midline and placed it at one of the four possible locations, identical to the target locations from Experiment 2 and the cue locations from Experiment 1. After $1 \mathrm{sec}$, the index finger was moved back to start position. Then the participants were instructed to point to the remembered location of their finger as accurately as possible. They confirmed verbally when they reached this location. The experimenter recorded the coordinates of the fingertip and moved the participants' finger back to the start position. Trials for each of the four target position were grouped in blocks. The target was presented six times within one block. The order of the blocks was randomized. If $\mathrm{S} 1_{\mathrm{EYE}_{\mathrm{E}} \mathrm{CTBS}}$ affect perceived position of the left index finger at rest, but not during movement, then one would predict a pointing error in this experiment after $\mathrm{S} 1_{\mathrm{EYE}}{ }^{-\mathrm{CT}} \mathrm{TBS}$.

\section{TMS}

A standard 70-mm-diameter figure-of-eight coil centered over the stimulation site was fixed in place by a coil holder. The participant's head was restrained by a chin rest. We followed an identical procedure for locating $\mathrm{S}_{\mathrm{EYE}}$ as in previous studies conducted by Balslev and colleagues (Odoj \& Balslev, 2013; Balslev, Gowen, et al., 2011; Balslev \& Miall, 2008). S1 $1_{\mathrm{EYE}}$ was mapped in each participant in relation to the "motor hotspot" of the left hemisphere, which is the scalp projection of the primary motor cortex for the hand (M1). The motor hotspot was defined as the point of maximum evoked motor response in the first dorsal interosseous muscle of the right hand. The $S 1_{\mathrm{EYE}}$ site of stimulation was located at $3 \mathrm{~cm}$ posterior to the motor hotspot, measured on a line oriented at $45^{\circ}$ from the sagittal plane and perpendicular on the central sulcus.

Post hoc neuronavigation has showed that that this coil location targets an area in the postcentral gyrus, at MNI coordinates (mean $\pm S D$ : $-45 \pm 7,-32 \pm 7,58 \pm 9$; 
Figure 4. Systematic shift in the locus of attention after $\mathrm{S} 1_{\mathrm{EYE}}$-CTBS. The locus of attention was defined as the location with the largest benefit of the cue. In leftward gaze, there was a rightward cueing error after $\mathrm{S} 1_{\mathrm{EYE}}$-CTBS (A) at all cue locations. This error was specific to $\mathrm{S} 1_{\mathrm{EYE}}-\mathrm{cTBS}$ and did not occur after the stimulation of the control site P3 (C). Likewise, in rightward gaze, there was a leftward cueing error at all cue locations after $\mathrm{S} 1_{\mathrm{EYE}}$-CTBS (B) but not after P3-cTBS (D). All values with SEM. $* p<.05, * * p<.01$, paired-samples $t$ test.

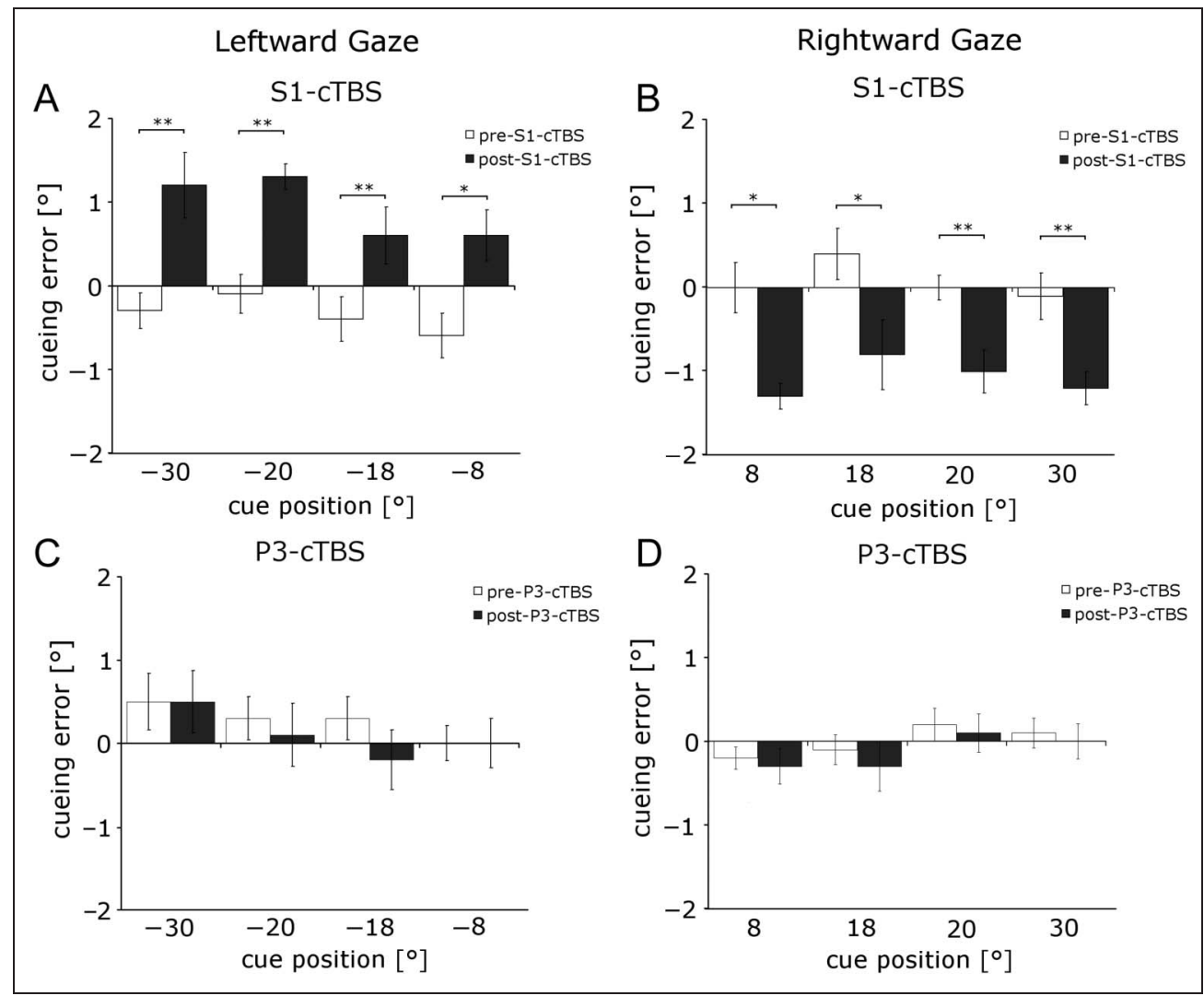

Balslev, Siebner, Paulson, \& Kassuba, 2012). The mean coordinate is associated with a probability of $46 \%$ for area $3 \mathrm{~b}, 37 \%$ for area 1 , and $27 \%$ for area 2 according to the probabilistic stereotaxic cytoarchitectonic atlas of the Anatomy Toolbox v2.1 (Eickhoff et al., 2005). The underestimation of the angle of gaze after $\mathrm{S} 1_{\mathrm{EYE}}$-CTBS for both left and right directions of gaze (Experiment 1 in Odoj \& Balslev, 2013) suggests that $S 1_{\mathrm{EYE}}$ is organized like the primary oculoproprioceptive area $3 \mathrm{a}$ in the macaque, where neurons encode gaze angle for all directions of gaze (Wang et al., 2007). It is unlikely that TMS applied over the scalp can reach area $3 a$, located in the depth of the central sulcus (Geyer, Schleicher, \& Zilles, 1999). The center-periphery principle of organization, however, may be common to all neural populations that receive an oculoproprioceptive projection. These neural populations are not limited to the depth of the central sulcus but extend into the postcentral and precentral gyri (Balslev, Albert, et al., 2011).

During stimulation, the coil was positioned tangential to the scalp with the long axis of the figure-of-eight coil oriented at $45^{\circ}$ to the parasagittal plane. The current flow of the initial rising phase of the biphasic pulse in the TMS coil induced a current flowing from posterior to anterior in the brain. On the basis of the decreased amplitude of the somatosensory-evoked potentials after cTBS over a region situated at $2 \mathrm{~cm}$ posterior to M1 (Ishikawa et al., 2007), we assumed that cTBS over $\mathrm{S} 1_{\mathrm{EYE}}$ results in a decreased excitability of this area. The control site of stimulation in the parietal lobe, P3, was located by using the International
10-20 system for EEG placement. Hilgetag and colleagues found that repetitive TMS over P3 improves visuospatial attention in the ipsilateral hemifield (Hilgetag, Theoret, \& Pascual-Leone, 2001). Therefore, we chose this area as control region to check if a possible effect, found in $\mathrm{S} 1_{\mathrm{EYE}}$-CTBS, could be explained by a spread of the induced current from $S 1_{\mathrm{EYE}}$ toward P3. cTBS consisted of 600 biphasic stimuli produced by a Magstim Rapid ${ }^{2}$ stimulator. They were delivered with a frequency of three pulses at $50 \mathrm{~Hz}$ repeated at $200 \mathrm{msec}(5 \mathrm{~Hz})$ for $40 \mathrm{sec}$. The stimulation intensity was set at $80 \%$ of active motor threshold of the right first dorsal interosseous (Huang, Edwards, Rounis, Bhatia, \& Rothwell, 2005). For each experiment, participants underwent two sessions, with cTBS at either $\mathrm{S} 1_{\mathrm{EYE}}$ or the control site (P3). The order of the sessions was randomized across participants and scheduled on separate days. During each session, the participant was tested before (pre-cTBS) and after (post-cTBS) on an identical task. Data collection was completed within 13 min after the cessation of the stimulation, a time interval for which the inhibitory aftereffect of cTBS in the somatosensory cortex has been demonstrated (Ishikawa et al., 2007).

\section{Eye Tracking}

The position of the right eye was recorded with a head mounted tracker that sampled pupil location at $250 \mathrm{~Hz}$. The tracker was calibrated after each cTBS run (pre- or post-cTBS) using a $3 \times 3$ grid. 
Eye position time series were parsed into fixations, blinks, and saccades using the SR EyeLink detection algorithm, which was set to detect saccades with an amplitude of at least $0.5^{\circ}$, using an acceleration threshold of $9500 \% \mathrm{sec}^{2}$ and a velocity threshold of $30 \% \mathrm{sec}$ and then analyzed offline. Trials with a mean deviation of more than $1.5^{\circ}$ from fixation within $50 \mathrm{msec}$ before target presentation were discarded. In Experiment 1, a total of $10.8 \pm 7.7 \%$ (mean $\pm S D$ ) trials were discarded. In Experiment 2, a total of $5.44 \pm 5.28 \%$ trials were discarded.

\section{RESULTS}

\section{Systematic Shift in the Locus of Attention Relative to the Cue after $S 1_{E Y E}$-cTBS}

Experiment 1 investigated whether oculoproprioception contributes to locating a nonvisual cue relative to a visual target in a cross-modal attention task. We predicted that, if this is the case, $\mathrm{S} 1_{\mathrm{EYE}}$-CTBS would shift the locus of attention to reflect the bias in the eye proprioceptive signal. In accord with the prediction, after cTBS over $\mathrm{S} 1_{\mathrm{EYE}}$ we found a cueing error of $0.6^{\circ}-1.3^{\circ}$ toward the midline (to the right in leftward gaze and to the left in rightward gaze; Figures 4A, B and 6A, B). The cueing error in the post $\mathrm{S} 1_{\mathrm{EYE}}$-CTBS run was significantly different from the pre-cTBS run at all tested cue locations (paired-samples $t$ tests: all $p s<.03)$. cTBS over P3 did not significantly change cueing error (Figure $4 \mathrm{C}$ and D, paired-samples $t$ test: all $p s>$.32). The repeated-measures ANOVA of mean cueing error with factors cTBS run (pre vs. post) $\times$ Stimulation area $\left(\mathrm{S} 1_{\mathrm{EYE}}\right.$ vs. P3) $\times$ Gaze direction (left vs. right) showed a significant three-way interaction $(F(1$, $9)=128.87, p<.001)$. Both post $\mathrm{S} 1_{\mathrm{EYE}^{-\mathrm{CTBS}}}$ values were significantly different from pre $\mathrm{S} 1_{\mathrm{EYE}}-\mathrm{C}$ TBS data (post hoc pairwise multiple comparison using Tukey's test, both $p s<.01)$. In comparison, there was no significant change in cueing error after control cTBS over P3 (both $p s>.05$ ).

A significant interaction was found for Gaze direction $\times$ cTBS run $(F(1,9)=72.6, p<.001)$. This interaction was driven by the post-S1 $1_{\mathrm{EYE}}$-CTBS effect in different directions for left and right gaze angles (post hoc pairwise multiple comparison using Tukey's test: $p<.01$ for left post-cTBS vs. right post-cTBS, all other $p$ s $>.05)$. None of the other main effects or interactions was significant (all $p s>.231$ ).

This result was robust across two different methods for identifying the locus of attention. We found the same effect of $S 1_{\mathrm{EYE}^{-}}$-CTBS when the locus of attention was calculated as the center of mass of the locations that showed a cueing benefit. After cTBS over $\mathrm{S} 1_{\mathrm{EYE}}$ cueing error was $0.4-1.2^{\circ}$ toward center (to the right in leftward gaze and to the left in rightward gaze; Figure $5 \mathrm{~A}$ and $\mathrm{B}$ ). The cueing error in the post $\mathrm{S} 1_{\mathrm{EYE}^{-}} \mathrm{CTBS}$ run was significant different from the pre-cTBS run at all tested cue locations (paired-samples $t$ tests: all $p s<.04$ ). cTBS over P3 did not change cueing error (Figure 5C and D,
Figure 5. Systematic shift in the locus of attention after $\mathrm{S} 1_{\mathrm{EYE}} \mathrm{C}^{\mathrm{CTBS}}$. The locus of attention was defined as the center of mass of all locations that showed a benefit of the cue. In leftward gaze, there was a rightward cueing error after $\mathrm{S} 1_{\mathrm{EYE}}$-CTBS $^{-\mathrm{A})}$ at all cue locations. This error was specific to $\mathrm{S} 1_{\mathrm{EYE}}-\mathrm{CTBS}$ and did not occur after the stimulation of the control site P3 (C). Likewise, in rightward gaze, there was a leftward cueing error at all cue locations after $S 1_{\text {EYE }}$-CTBS (B) but not after P3-cTBS (D). All values with SEM. $* p<.05, * * p<.01$, $* * * p<.001$ in paired-samples $t$ test.

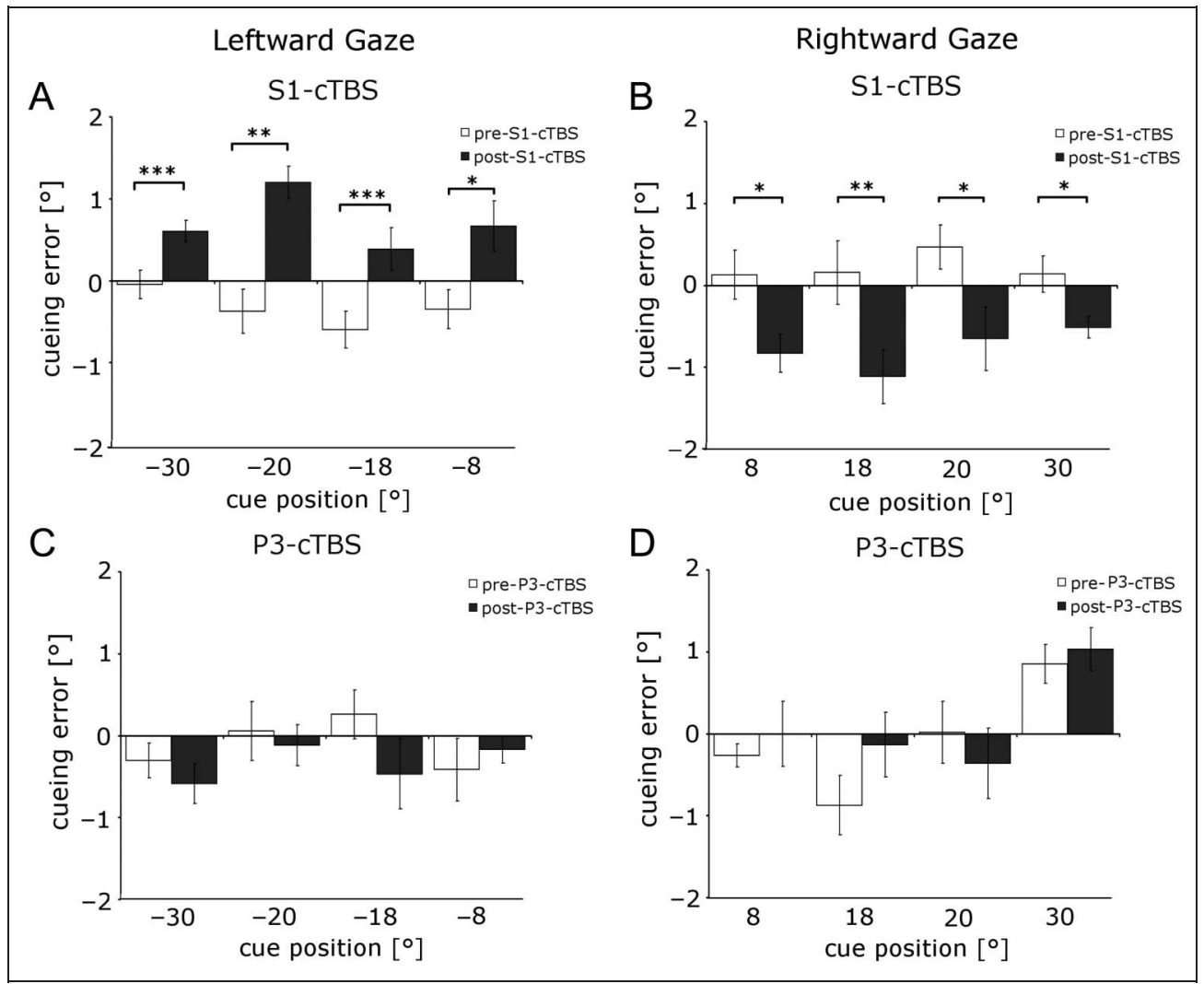


paired-samples $t$ test: all $p s>.21)$. Repeated-measures ANOVA with factors cTBS run (pre vs. post) $\times$ Stimulation area $\left(\mathrm{S}_{\mathrm{EYE}}\right.$ vs. $\left.\mathrm{P} 3\right) \times$ Gaze direction (left vs. right) showed a significant three-way interaction $(F(1,9)=$ $45.9, p<.001)$. For leftward gaze, the mean cueing error was $-0.34 \pm 0.3^{\circ}$ (pre-S1 $1_{\mathrm{EYE}}$-CTBS) and $-0.1 \pm 0.4^{\circ}$ (pre$\mathrm{P} 3$-cTBS). For rightward gaze, these values were $0.2 \pm$ $0.52^{\circ}$ for pre $S 1_{\mathrm{EYE}^{-\mathrm{C}} \mathrm{CBS}}$ and $-0.07 \pm 0.46^{\circ}$ for pre P3cTBS. After $\mathrm{S} 1_{\mathrm{EYE}} \mathrm{-CTBS}$, the cueing error was $0.71 \pm$ $0.51^{\circ}$ in leftward gaze and $-0.78 \pm 0.42^{\circ}$ in rightward gaze. Both post $\mathrm{S} 1_{\mathrm{EYE}}$-c $\mathrm{TBS}$ values were significantly different from pre $S 1_{\mathrm{EYE}^{-\mathrm{C}}} \mathrm{TBS}$ data (post hoc pairwise multiple comparison using Tukey's test, both $p s<.02)$.

Here as well in the previous analysis, a significant interaction was found for Gaze direction $\times$ cTBS run $(F(1,9)=$ $19.1, p<.001)$, driven by the post-S1 $1_{\mathrm{EYE}}-\mathrm{CTBS}$ effect in different directions for left and right gaze angles (post hoc pairwise multiple comparison using Tukey's test: $p<.01$ for left post-cTBS vs. right post-cTBS, all other $p s>.05)$. None of the other main effects and interactions was statistically significant (all $p s>.115$ ). After P3-cTBS, no significant shift in cueing error could be observed (mean cueing error $-0.33 \pm 0.56^{\circ}$ for screen left, $0.13 \pm$ $0.52^{\circ}$ for screen right, post hoc pairwise multiple comparison using Tukey's test: both $p s>.05$ ).

Participants' accuracy was close to ceiling. They were correct in $97.85 \pm 1.73 \%$ of the trials. We found no differ- ence in accuracy across conditions. Repeated-measures ANOVA with factors cTBS run (pre vs. post) $\times$ Stimulation area $\left(\mathrm{S}_{\mathrm{EYE}}\right.$ vs. $\left.\mathrm{P} 3\right) \times$ Gaze direction (left vs. right) showed no significant three-way interaction $(F(1,9)=.276, p=$ $.612)$. None of the main effects or two-way interactions was significant (all $p s>.272$ ).

\section{No Change in Open-loop Pointing to Visual Targets After S1 EYE$_{\text {-cTBS }}$}

Experiment 2 investigated whether $S 1_{\mathrm{EYE}}$-CTBS impacts the perceived location of visual objects or perceived posture of left hand. If this was the case, one would expect an increase in pointing error after $\mathrm{S} 1_{\mathrm{EYE}}{ }^{-\mathrm{C}} \mathrm{TBS}$.

We did not find a statistically significant increase in pointing error for any target position, gaze direction, or stimulation site (Figure 6, paired-samples $t$ tests pre- vs. poststimulation, all $p s>.102$ ). The mean pointing error over all target locations did not change significantly after cTBS (Figure 7C, D). The repeated-measures ANOVA of the pointing error with factors TMS run (pre vs. post) $\times$ Stimulation area ( $\mathrm{S}_{\mathrm{EYE}} \mathrm{vs}$. P3) $\times$ Gaze direction (left vs. right) showed no significant three-way interaction $(F(1$, $9)=1.33, p>.27$ ), main effects or two-way interactions (all $p s>$.112).

The precision of pointing was not changed either by cTBS either. The repeated-measures ANOVA with factors
Figure 6. No shift in visual pointing for any cueing position after $\mathrm{S}_{\mathrm{EYE}^{-}}$or P3-cTBS. In leftward gaze, there was a rightward pointing error after $\mathrm{S} 1_{\mathrm{EYE}}$-CTBS (A). This error was specific to $\mathrm{S} 1_{\mathrm{EYE}}$-cTBS and did not occur after the stimulation of the control site P3 (C). In rightward gaze, there was a leftward pointing error only after $\mathrm{S} 1_{\mathrm{EYE}}$-cTBS (B) but not after P3-cTBS (D). All values with SEM.

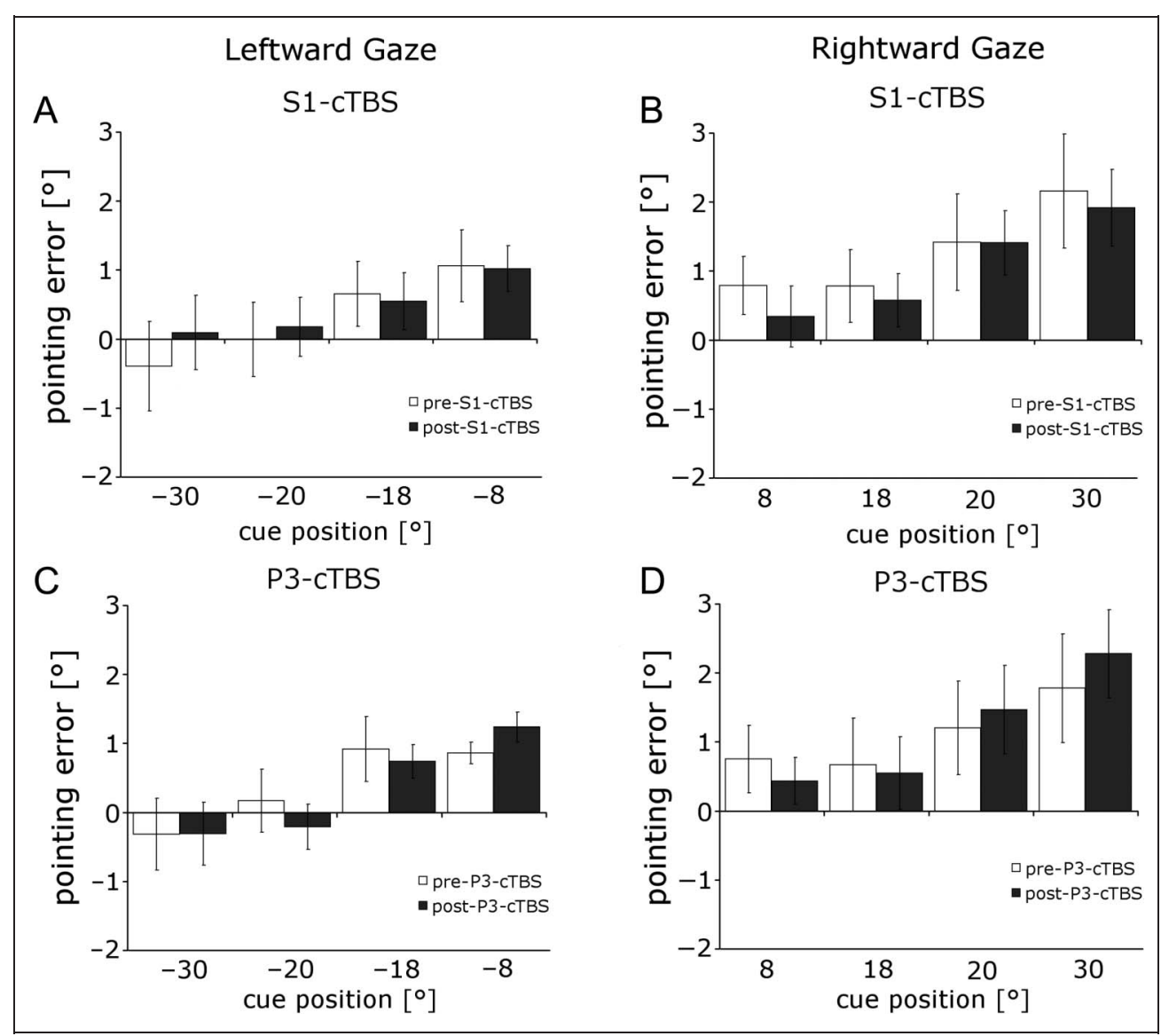


Figure 7. The effect of $\mathrm{S} 1_{\mathrm{EYE}}-\mathrm{CTBS}$ is specific for coding the locus of attention. Upper row shows the mean cueing error in the cross-modal attention task for leftward (A) and rightward (B) gaze. The middle row shows the mean error for open-loop pointing to visual targets $(C, D)$, and the lower row shows the pointing error to somatosensory targets (E, F). In the left column, data for left gaze direction are shown; the right column shows data for right gaze direction. Filled circles ( show pre-cTBS data; empty circles $(O)$ show post-cTBS data Only changes in cueing error after $\mathrm{S}_{\mathrm{EYE}}$-cTBS were significant. All values with $1 S D$. **p $<.01$ in post hoc multiple comparison using Tukey's test.

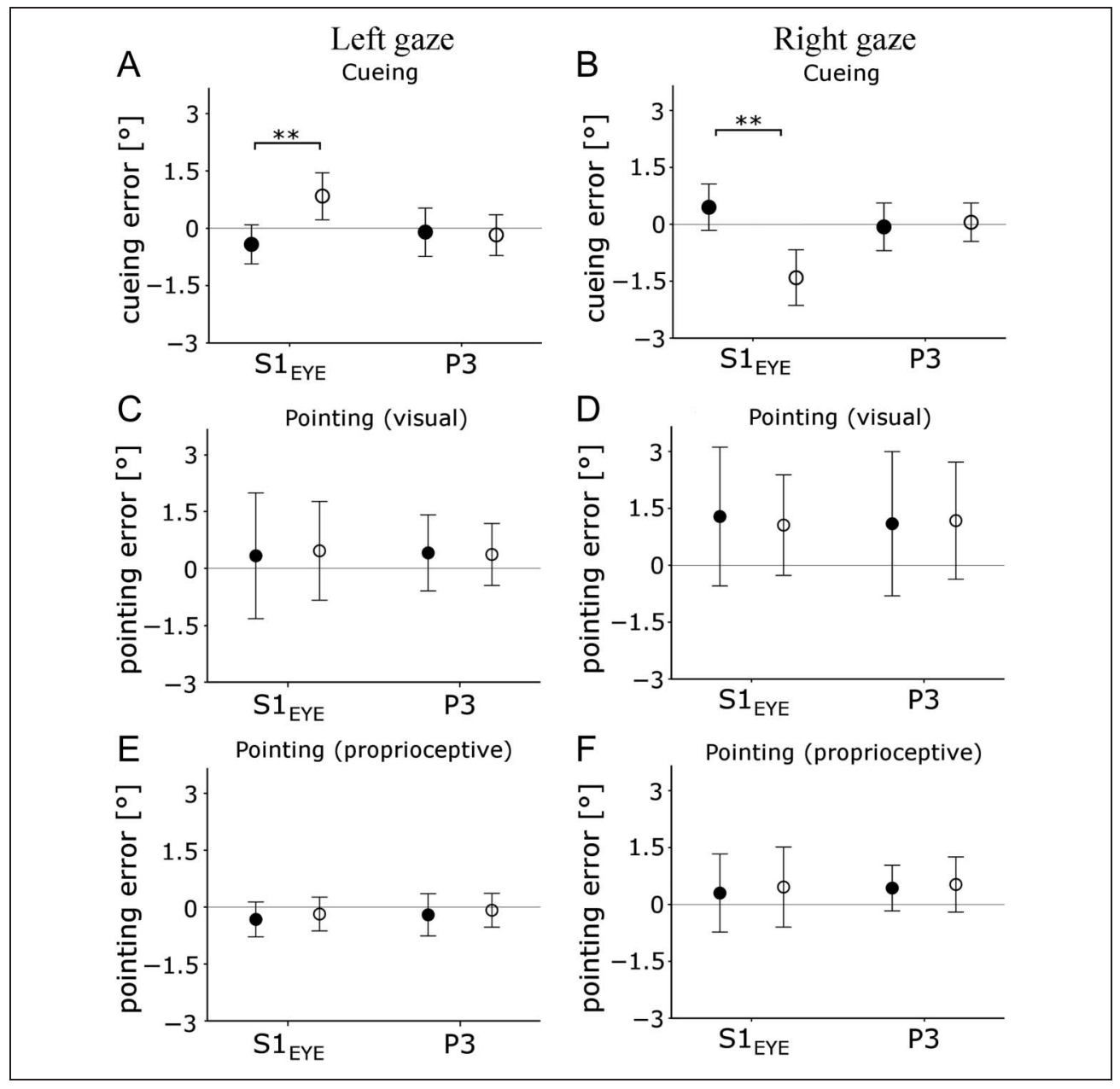

TMS run (pre vs. post) $\times$ Stimulation area $\left(S 1_{\mathrm{EYE}}\right.$ vs. P3) $\times$ Gaze direction (left vs. right) for the $S D$ of pointing error showed no significant three-way interaction $(F(1,9)$ $=0.98, p>.32$ ) and no significant main effects or twoway interactions (all $p s>.181$ ). For left gaze direction, the mean $\pm S D$ of the pointing error across participants was $0.82 \pm 0.44^{\circ}$ before $S 1_{\mathrm{EYE}^{-} \mathrm{CTBS}}$ and $0.75 \pm 0.50^{\circ}$ after $\mathrm{S} 1_{\mathrm{EYE}}{ }^{-\mathrm{CTBS}}$. The values for P3-cTBS were $1.15 \pm 48^{\circ}$ (precTBS) and $1.01 \pm 0.35^{\circ}$ (post c-TBS). For right gaze direction, participants had $S D$ s of $1.83 \pm 0.97^{\circ}$ before $S 1_{\mathrm{EYE}^{-}}$ cTBS and $1.36 \pm 0.86^{\circ}$ after $\mathrm{S} 1_{\mathrm{EYE}^{-}} \mathrm{cTBS}$. The P3-cTBS values were $1.53 \pm 0.84^{\circ}$ (pre-cTBS) and $1.49 \pm 0.93^{\circ}$ (post-cTBS).

Direct comparison between the cueing and pointing error in Experiments 1 and 2 using a repeated-measures ANOVA with factors (1) Task (cross-modal attention vs. pointing), (2) TMS run (pre vs. post), (3) Stimulation area (S1 $1_{\mathrm{EYE}}$ vs. $\mathrm{P} 3$ ), and (4) Gaze direction (leftward vs. rightward) showed a statistically significant four-way interaction $(F(1,9)=13.327, p<.008)$. No significant main effects were found (all $p s>.105$ ). All significant interactions (Task $\times$ Gaze direction: $F(1,9)=9.99, p=.012$; TMS run $\times$ Gaze direction: $F(1,9)=13.14, p=.006$; task $\times$ TMS run $\times$ Gaze direction: $F(1,9)=29.67, p<.001$; TMS run $\times$ Stimulation area $\times$ Gaze direction: $F(1,9)=$
$27.18, p=.001$ ) were driven by opposite effect of $S 1_{\mathrm{EYE}^{-}} \mathrm{CTBS}$ for different directions of gaze in the crossmodal attention task (post hoc pairwise multiple comparison using Tukey's test: $p<.01$ for left post-cTBS vs. right post-cTBS and for left post-cTBS vs. right post-cTBS, all other $p s>.05)$. A post hoc Tukey's test, comparing pre- vs. post-cTBS, was only significant for pre- vs. post-S1 $1_{\mathrm{EYE}_{\mathrm{E}}-\mathrm{CTBS}}$ in the cross-modal attention task for either gaze direction.

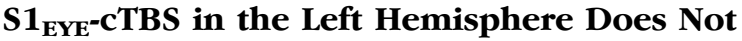 Disturb Perceived Position of the Ipsilateral, Left Index Finger at Rest}

Experiment 3 controlled for an effect of $\mathrm{S} 1_{\mathrm{EYE}}$-CTBS on felt position of the left index finger at rest. If perceived finger position at rest, but not during pointing, is disturbed by cTBS over $\mathrm{S} 1_{\mathrm{EYE}}$, one would expect an increase in pointing error after $\mathrm{S}_{\mathrm{EYE}_{\mathrm{E}}} \mathrm{c} \mathrm{TBS}$ here.

We found no significant change in pointing error between pre- and post-cTBS over $\mathrm{S}_{\mathrm{EYE}}$ or $\mathrm{P} 3$ for any of the eight tested locations (paired-samples $t$ tests: all $p s>.106$ ). Repeated-measures ANOVA of mean pointing errors with factors Gaze direction (left vs. right) $\times$ Stimulation site $\left(\mathrm{S} 1_{\mathrm{EYE}}\right.$ vs. P3) $\times$ Run (pre vs. post) showed neither main effects nor interactions (all $p s>.89$ ). 
The mean pointing error for each participant before and after CTBS over $\mathrm{S}_{\mathrm{EYE}}$ or $\mathrm{P} 3$ is shown in Figure $7 \mathrm{E}, \mathrm{F}$.

\section{DISCUSSION}

We found that altering the activity of an oculoproprioceptive area in the human postcentral gyrus is sufficient to divert attention away from behaviorally important cues. Throughout the visual space, cueing error, or the distance between the cue and the locus of attention, matched well in direction and magnitude the bias in perceived eye rotation measured in a previous study (Odoj $\&$ Balslev, 2013). This error cannot be explained by a mislocalization of the visual target. Direct comparison between the attention and reaching tasks (Experiments 1 and 2) showed a significant interaction, driven by the larger error for cross-modal attention than for visually guided reaching. We argue therefore that oculoproprioception is selective for the attention map, as opposed to the visual map for reaching.

Because oculoproprioception was manipulated at cortical level, one could object that the effect of cTBS on attention was merely the result of the disruption of the cortical modules dedicated to this function. This explanation is unlikely. The direction of the cueing error was gaze dependent (leftward in right gaze and rightward in left gaze), and its magnitude at all tested locations matched well the error in perceived eye position (Odoj \& Balslev, 2013).

\section{A Role of Oculoproprioception in Coding the Locus of Attention Is Compatible with Neurophysiological Data}

Two neural mechanisms have been proposed to implement a locus of attention that anchors retinotopic representations to the physical location of the visual stimuli. First, populations of gain-field neurons with a retinotopic receptive field scale their activity with the angle of gaze to encode visual location relative to the body and/or the world (Pouget et al., 2002; Andersen \& Mountcastle, 1983). A second mechanism is remapping of the retinotopic receptive field to account for eye movements (Mirpour \& Bisley, 2012; Duhamel et al., 1992). A role of oculoproprioception in coding the locus of attention is compatible with both these mechanisms. A signature of the fastest of the two components of the oculoproprioceptive feedback can be recorded from somatosensory area 3a already at $5 \mathrm{msec}$ before the end of the saccade (Wang et al., 2007). This is in line with the time needed for a remapped representation for the locus of attention to emerge in area LIP at 8-32 msec after the saccade (Mirpour \& Bisley, 2012). Likewise, gain-field neurons show $\sim 150$ msec postsaccadic delay in updating (Xu, Karachi, \& Goldberg, 2012), which is compatible with the $\sim 60$ msec latency of the slower, tonic (Xu et al., 2011) component of the oculoproprioceptive signal.

\section{Implications of the Current Findings for Understanding the Coupling between the Eye and Attention}

Although the idea that the eye and attention systems are coupled is not new (Rizzolatti, Riggio, Dascola, \& Umiltá, 1987), the mechanism of this coupling remains a current topic of debate (Smith \& Schenk, 2012; Wright \& Ward, 2008). We argue here that the attention maps incorporate oculosensory signals to align retinotopic representations to physical locations. A role of the oculosensory signals in spatial attention is also suggested by observations of a reduced ability to orient attention to cues in patients suffering from a disease of the extraocular muscles or their peripheral innervation (Gabay, Henik, \& Gradstein, 2010; Smith, Rorden, \& Jackson, 2004; Craighero, Carta, \& Fadiga, 2001). Under the assumption that in these patients the brain areas that relay the corollary discharge (Sommer \& Wurtz, 2008) are normal, such observations can provide insight into the role of the oculoproprioceptive inflow in spatial attention. Likewise, the allocation of attention is disrupted at extreme rotations of the eyes (Smith, Schenk, \& Rorden, 2012; Smith, Ball, Ellison, \& Schenk, 2010; Craighero, Nascimben, \& Fadiga, 2004), a condition known to cause an abnormal oculoproprioceptive inflow (Paap \& Ebenholtz, 1976; Ebenholtz, 1974).

Smith and Schenk have proposed that the oculomotor signals bias the competition among sensory inputs to favor stimuli that are also the end point of a planned saccade (Smith \& Schenk, 2012). In line with the idea that attention follows the gaze, can an alternative explanation of the current results be that oculoproprioception merely biases perception to favor visual stimuli nearer the perceived direction of gaze? The design of the spatial attention task rules out this explanation. This is because an unspecific bias toward perceived direction of gaze would have been identical during both conditions, with and without a cue. $S 1_{\mathrm{EYE}}{ }^{-\mathrm{CTBS}}$ changed the difference in $\mathrm{RT}$ between these two conditions. Thus, cueing error after $\mathrm{S} 1_{\mathrm{EYE}}$-CTBS cannot be explained by a general bias in spatial attention toward the midline.

\section{Can the Selective Role of Oculoproprioception in Spatial Attention Be Explained by the Timing of the Underlying Neural Processing?}

Our result is surprising. We found that participants match the same hand location with different retinal locations depending on whether the hand acts as an attention cue or as an effector for action. These results cannot be explained by differences between the attention and pointing task in the timing of the visual target onset. Because of the delay in the ascending pathways, 
oculoproprioception can be unreliable for up to $60 \mathrm{msec}$ after an eye movement (Xu et al., 2011; Wang et al., 2007). In both tasks, participants maintained fixation for 600-750 msec before visual target onset. So, it is safe to assume that, at target onset, reliable oculoproprioception had been available. The lack of a detectable effect of the oculoproprioceptive distortion on visually guided reaching is in line with previous findings. Some studies fail to find errors in locating visual targets relative to the body when oculoproprioception is reduced or abnormal (Balslev, Himmelbach, et al., 2012; Lewis et al., 1998) or calculate a smaller weight for oculoproprioception than for the corollary discharge in the multimodal estimate of eye position (Bridgeman \& Stark, 1991; Gauthier, Nommay, \& Vercher, 1990).

We speculate that the reason for the larger weight of oculoproprioceptive signal from the somatosensory cortex for spatial attention rather than for visually guided reaching is the timing of the underlying neural processes. If neural processing necessary to build attention representations exceeds the time interval in which proprioception is unreliable and if the neural processing for the visual map does not, then only the former would incorporate oculoproprioception. In support of this idea, brain areas that process shape or color (i.e., mainly dedicated to perceptual discrimination) have a longer latency of the visually evoked activity compared with brain areas that process luminance or motion (i.e., mainly for action; Laycock, Crewther, \& Crewther, 2007; Schmolesky et al., 1998; Schroeder, Mehta, \& Givre, 1998). The longer latency of neural processing for perception versus action may leave more time for computing priority versus reaching maps. The forward model based on the corollary discharge provides an early estimate of eye position that is probably reasonably accurate, given the predictable environment of the orbits. Therefore, fast neural processes (i.e., computing visual maps for reaching) are likely to rely on corollary discharge or, alternatively, on oculoproprioceptive input available upstream the somatosensory cortex. Subcortical structures that could provide oculoproprioceptive signals for visually guided reaching are the superior colliculus, which in rats is connected to the trigeminal nucleus (Ndiaye, Pinganaud, VanderWerf, Buisseret-Delmas, \& Buisseret, 2000) or the central thalamus that in the monkey contains neurons sensitive to eye position which discharge after a saccade (Tanaka, 2007). In contrast, neural processes that take longer (i.e., computing the priority map for perception) may accommodate the delay in the ascending proprioceptive pathways and benefit from a more robust estimate of eye position by incorporating the oculoproprioceptive input from the somatosensory cortex.

\section{Acknowledgments}

This work was supported by the Danish Medical Research Councils (grant number 09-072209 to D. B.). We thank Prof.
H.-O. Karnath for hosting these experiments in the Section of Neuropsychology at the University of Tübingen.

Reprint requests should be sent to Daniela Balslev, School of Psychology and Neuroscience, University of St. Andrews, St Mary's Quad, South Street, St. Andrews, KY16 9JP, United Kingdom, or via e-mail: daniela.balslev@st-andrews.ac.uk.

\section{REFERENCES}

Andersen, R. A., \& Mountcastle, B. (1983). The influence of the angle of gaze upon the excitability of the light-sensitive neurons of the posterior parietal cortex. Journal of Neuroscience, 3, 532-548.

Andersen, R. A., Snyder, L. H., Bradley, D. C., \& Xing, J. (1997). Multimodal representation of space in the posterior parietal cortex and its use in planning movements. Annual Review of Neuroscience, 20, 303-330.

Balslev, D., Albert, N. B., \& Miall, R. C. (2011). Eye muscle proprioception is represented bilaterally in the sensorimotor cortex. Human Brain Mapping, 32, 624-631.

Balslev, D., Gowen, E., \& Miall, R. C. (2011). Decreased visual attention further from the perceived direction of gaze for equidistant retinal targets. Journal of Cognitive Neuroscience, 23, 661-669.

Balslev, D., Himmelbach, M., Karnath, H.-O., Borchers, S., \& Odoj, B. (2012). Eye proprioception used for visual localization only in conflict with the oculomotor plan. Journal of Neuroscience, 32, 8569-8573.

Balslev, D., Siebner, H. R., Paulson, O. B., \& Kassuba, T. (2012). The cortical eye proprioceptive signal modulates neural activity in higher-order visual cortex as predicted by the variation in visual sensitivity. Neuroimage, 61 , 950-956.

Balslev, D., \& Miall, R. C. (2008). Eye position representation in human anterior parietal cortex. Journal of Neuroscience, 28, 8968-8972.

Balslev, D., Newman, W., \& Knox, P. C. (2012). Extraocular muscle afferent signals modulate visual attention. Investigative Ophthalmology \& Visual Science, 53, 7004-7009.

Balslev, D., Odoj, B., \& Karnath, H.-O. (2013). Role of somatosensory cortex in spatial attention. Journal of Neuroscience, 33, 18311-18318.

Bridgeman, B., \& Stark, L. (1991). Ocular proprioception and efference copy in registering visual direction. Vision Research, 31, 1903-1913.

Craighero, L., Carta, A., \& Fadiga, L. (2001). Peripheral oculomotor palsy affects orienting of visuospatial attention. NeuroReport, 12, 3283-3286.

Craighero, L., Nascimben, M., \& Fadiga, L. (2004). Eye position affects orienting of visuospatial attention. Current Biology, 14, 331-333.

Duhamel, J. R., Colby, C. L., \& Goldberg, M. E. (1992). The updating of the representation of visual space in parietal cortex by intended eye movements. Science, 255, 90-92.

Durand, J.-B., Camors, D., Trotter, Y., \& Celebrini, S. (2012). Privileged visual processing of the straight-ahead direction in humans. Journal of Vision, 12, 34.

Durand, J.-B., Trotter, Y., \& Celebrini, S. (2010). Privileged processing of the straight-ahead direction in primate area V1. Neuron, 66, 126-137.

Ebenholtz, S. M. (1974). The possible role of eye-muscle potentiation in several forms of prism adaptation. Perception, 3, 477-485.

Eickhoff, S. B., Stephan, K. E., Mohlberg, H., Grefkes, C., Fink, G. R., Amunts, K., et al. (2005). A new SPM toolbox for combining probabilistic cytoarchitectonic maps and functional imaging data. Neuroimage, 25, 1325-1335. 
Gabay, S., Henik, A., \& Gradstein, L. (2010). Ocular motor ability and covert attention in patients with Duane Retraction Syndrome. Neuropsychologia, 48, 3102-3109.

Gauthier, G. M., Nommay, D., \& Vercher, J. L. (1990). The role of ocular muscle proprioception in visual localization of targets. Science, 249, 58-61.

Geyer, S., Schleicher, A., \& Zilles, K. (1999). Areas 3a, 3b, and 1 of human primary somatosensory cortex. Neuroimage, $10,63-83$.

Golomb, J. D., Chun, M. M., \& Mazer, J. A. (2008). The native coordinate system of spatial attention is retinotopic. Journal of Neuroscience, 28, 10654-10662.

Golomb, J. D., Nguyen-Phuc, A. Y., Mazer, J. A., McCarthy, G., \& Chun, M. M. (2010). Attentional facilitation throughout human visual cortex lingers in retinotopic coordinates after eye movements. Journal of Neuroscience, 30, 10493-10506.

Gottlieb, J. P., Kusunoki, M., \& Goldberg, M. E. (1998). The representation of visual salience in monkey parietal cortex. Nature, 391, 481-484.

Grubb, J. D., Reed, C. L., Bate, S., Garza, J., \& Roberts, R. L. (2008). Walking reveals trunk orientation bias for visual attention. Perception \& Psychophysics, 70, 688-696.

Guthrie, B. L., Porter, J. D., \& Sparks, D. L. (1983). Corollary discharge provides accurate eye position information to the oculomotor system. Science, 221, 1193-1195.

Hilgetag, C. C., \& Pascual-Leone, A. (2001). Enhanced visual spatial attention ipsilateral to rTMS-induced "virtual lesions" of human parietal cortex Nature Neuroscience, 4, 953-957.

Huang, Y. Z., Edwards, M. J., Rounis, E., Bhatia, K. P., \& Rothwell, J. C. (2005). Theta burst stimulation of the human motor cortex. Neuron, 45, 201-206.

Ishikawa, S., Matsunaga, K., Nakanishi, R., Kawahira, K., Murayama, N., Tsugi, S., et al. (2007). Effect of theta burst stimulation over the human sensorimotor cortex on motor and somatosensory evoked potentials. Clinical Neurophysiology, $118,1033-1043$.

Laycock, R., Crewther, S. G., \& Crewther, D. P. (2007). A role for the "magnocellular advantage" in visual impairments in neurodevelopmental and psychiatric disorders. Neuroscience and Biobehavioral Reviews, 31, 363-376.

Lewis, R. F., Gaymard, B. M., \& Tamargo, R. J. (1998). Efference copy provides the eye position information required for visually guided reaching. Journal of Neurophysiology, 80, 1605-1608.

Mathôt, S., \& Theeuwes, J. (2010). Gradual remapping results in early retinotopic and late spatiotopic inhibition of return. Psychological Science, 21, 1793-1798.

Mirpour, K., \& Bisley, J. W. (2012). Anticipatory remapping of attentional priority across the entire visual field. Journal of Neuroscience, 32, 16449-16457.

Ndiaye, A., Pinganaud, G., VanderWerf, F., Buisseret-Delmas, C., \& Buisseret, P. (2000). Connections between the trigeminal mesencephalic nucleus and the superior colliculus in the rat. Neuroscience Letters, 294, 17-20.

Odoj, B., \& Balslev, D. (2013). Visual sensitivity shifts with perceived eye position. Journal of Cognitive Neuroscience, 25, 1180-1189.

Paap, K. R., \& Ebenholtz, M. (1976). Perceptual consequences of potentiation in the extraocular muscles: An alternative explanation for adaptation to wedge prisms. Journal of Experimental Psychology: Human Perception and Performance, 2, 457-468.

Pouget, A., Deneve, S., \& Duhamel, J. R. (2002). A computational perspective on the neural basis of multisensory spatial representations. Nature Reviews Neuroscience, 3, 741-747.
Reed, C. L., Grubb, J. D., \& Steele, C. (2006). Hands up: Attentional prioritization of space near the hand. Journal of Experimental Psychology: Human Perception and Performance, 32, 166-177.

Rizzolatti, G., Riggio, L., Dascola, I., \& Umiltá, C. (1987). Reorienting attention across the horizontal and vertical meridians: Evidence in favor of a premotor theory of attention. Neuropsychologia, 25, 31-40.

Schmolesky, M. T., Wang, Y., Hanes, D. P., Thompson, K. G., Leutgeb, S., Schall, J. D., et al. (1998). Signal timing across the macaque visual system. Journal of Neurophysiology, 79, 3272-3278.

Schroeder, C. E., Mehta, A. D., \& Givre, S. J. (1998). A spatiotemporal profile of visual system activation revealed by current source density analysis in the awake macaque. Cerebral Cortex, 8, 575-592.

Sherrington, C. S. (1918). Observations on the sensual role of the proprioceptive nerve-supply of the extrinsic ocular muscles. Brain, 41, 332-343.

Smith, D. T., Ball, K., Ellison, A., \& Schenk, T. (2010). Deficits of reflexive attention induced by abduction of the eye. Neuropsychologia, 48, 1269-1276.

Smith, D. T., Rorden, C., \& Jackson, S. R. (2004). Exogenous orienting of attention depends upon the ability to execute eye movements. Current Biology, 14, 792-795.

Smith, D. T., \& Schenk, T. (2012). The premotor theory of attention: Time to move on? Neuropsychologia, 50, 1104-1114.

Smith, D. T., Schenk, T., \& Rorden, C. (2012). Saccade preparation is required for exogenous attention but not endogenous attention or IOR. Journal of Experimental Psychology. Human Perception and Performance, $38,1438-1447$.

Sommer, M. A., \& Wurtz, R. H. (2008). Brain circuits for the internal monitoring of movements. Annual Review of Neuroscience, 31, 317-338.

Steinbach, M. J. (1986). Inflow as a long-term calibrator of eye position in humans. Acta Psychologica, 63, 297-306.

Talsma, D., White, B. J., Mathôt, S., Munoz, D. P., \& Theeuwes, J. (2013). A retinotopic attentional trace after saccadic eye movements: Evidence from event-related potentials. Journal of Cognitive Neuroscience, 25, 1563-1577.

Tanaka, M. (2007). Spatiotemporal properties of eye position signals in the primate central thalamus. Cerebral Cortex, 17, 1504-1515.

von Helmholtz, H. (1925). In J. P. C. Southhall (Ed.), Helmboltz's treatise on physiological optics (Vol. 3, pp. 242-270). New York: Optical Society of America, Electronic edition (2001): (pp. 242-270). University of Pennsylvania.

Wang, X., Zhang, M., Cohen, I. S., \& Goldberg, M. E. (2007). The proprioceptive representation of eye position in monkey primary somatosensory cortex. Nature Neuroscience, 10, 640-646.

Wright, R. D., \& Ward, L. M. (2008). Orienting of attention. New York: Oxford University Press.

Wurtz, R. H. (2008). Neuronal mechanisms of visual stability. Vision Research, 48, 2070-2089.

$\mathrm{Xu}$, B. Y., Karachi, C., \& Goldberg, M. E. (2012). The postsaccadic unreliability of gain fields renders it unlikely that the motor system can use them to calculate target position in space. Neuron, 76, 1201-1209.

Xu, Y., Wang, X., Peck, C., \& Goldberg, M. E. (2011). The time course of the tonic oculomotor proprioceptive signal in area 3a of somatosensory cortex. Journal of Neurophysiology, 106, 71-77. 